\title{
Framework de Persistência para Implementação De Aplicações Multi-tenant em Java
}

\author{
Matheus de A. Cordeiro ${ }^{1}$, Emanuell F. H. de Lucena ${ }^{1}$ \\ ${ }^{1}$ Faculdades Integradas de Patos (FIP) \\ 58.700-250 - Patos - PB - Brasil \\ \{matheus, emanuell\}@ffm.com.br
}

\begin{abstract}
The growing demand for cloud applications, due to high maintenance costs of the services offered by the traditional model of software offering, created the architectural model multi-tenancy, which allows the optimization of resources and infrastructure software systems sharing the same application instance and maintaining customer data logically separate. In the Java language, the JDBC API, widely used by the developer community, still does not natively support multi-tenant connections. This paper presents a framework that has these features to make possible the implementation of multi-tenant applications in Java using JDBC API for connecting to the database. At the beginning of this work, the methodology consists in performing literature searches on the central issue and related topics in order to acquire the necessary theoretical knowledge. Then, using the knowledge acquired in the previous step, are described the requirements and structure of a framework, applying the concepts previously seen. Finally, the creation and implementation of a case study using the framework presented.
\end{abstract}

Resumo. Com a crescente demanda de aplicações em nuvem, devido aos altos custos de manutenção dos serviços oferecidos pelo modelo tradicional de oferta de software, surge o modelo de arquitetura multi-tenancy, que permite a otimização de recursos de infraestrutura e sistemas de software compartilhando uma mesma instância de aplicação e mantendo os dados dos clientes separados de forma lógica. Na linguagem Java, a API JDBC, amplamente utilizada pela comunidade de desenvolvedores, ainda não dá suporte nativo a conexões multi-tenant. Este trabalho apresenta um framework que segue essas características para tornar possivel a implementação de aplicações multi-tenant em Java utilizando a API JDBC para conexão com o banco de dados. Nas primeiras etapas deste trabalho, a metodologia adotada consiste na realização de pesquisas bibliográficas sobre o assunto central e temas relacionados, com o intuito de adquirir o conhecimento teórico necessário. Em seguida, utilizando-se dos conhecimentos adquiridos na etapa anterior, são descritos os requisitos e estrutura de um framework, aplicando os conceitos vistos anteriormente. Por fim, a criação e execução de um estudo de caso utilizando o framework apresentado. 


\section{Introdução}

As aplicações de software tradicionais, também chamadas de stand-alone, são construídas e disponibilizadas seguindo o modelo de arquitetura single-tenant, no qual corresponde ao modelo de manutenção que utiliza-se de um conjunto de licenças contratuais e custos anuais de suporte para um cliente em questão, seguindo todas as especificações desejadas.

Do ponto de vista do fornecedor, a aplicação se torna específica para um único cliente, e qualquer expansão ou reuso para novos clientes, que buscam funcionalidades semelhantes, exige um grande esforço de remodelagem e desenvolvimento.

Com o surgimento da Cloud Computing, virtualização de produtos e serviços computacionais, tornou-se possível a adoção do modelo de arquitetura multi-tenancy, onde uma aplicação, hospedada em servidores na Internet, é compartilhada entre vários usuários distintos, de forma que estes acessem a mesma aplicação e tenham seus dados independentes dos demais usuários. Com isso, o acesso aos dados se torna restrito, eliminando o risco de acessos não-autorizados.

Ao contratar o serviço, o usuário pode acessá-lo a partir de um navegador $W e b$, economizando custos com licenciamento e recursos, tais como: pessoas, hardware, software e instalação. Neste modelo, o usuário paga apenas pelo que usar e os recursos da aplicação são de fácil expansão [Brito 2012].

Atualmente, a linguagem de programação Java já possui frameworks de conexão com banco de dados que podem possibilitar a separação necessária para a implementação de aplicações multi-tenant. Um framework é um conjunto de classes que incorporam um arcabouço para solucionar determinados problemas relacionados [Fayad et al. 1999]. Um dos frameworks Java mais populares é o Hibernate [Kabanov 2011]. Com ele, é possível realizar o mapeamento objeto-relacional de uma aplicação, o gerenciamento de conexões com o banco de dados, além do suporte à multi-tenancy.

Internamente o Hibernate utiliza-se de uma Application Programming Interface (API), que é um conjunto de classes e interfaces nativamente desenvolvidas, chamada de Java Database Connectivity (JDBC). Essa API tem a função de abstrair a forma de comunicação e envio das instruções da aplicação para o banco de dados. Todavia, a API JDBC ainda não dá suporte nativo à conexões multi-tenant. Por ser muito utilizada pela comunidade de desenvolvedores Java, é interessante que haja uma solução para esse novo modelo de aplicações o mais próximo da API nativa.

Portanto, o objetivo deste trabalho é construir um framework para implementação de multi-tenancy em aplicações Java que utilizam a API JDBC para conexão com banco de dados.

Nas primeiras etapas deste trabalho, a metodologia adotada consiste na realização de pesquisas bibliográficas sobre o assunto central e temas relacionados, com o intuito de adquirir o conhecimento teórico necessário. Em seguida, utilizando-se dos conhecimentos adquiridos na etapa anterior, são descritos os requisitos e estrutura de um framework, aplicando os conceitos vistos anteriormente. Por fim, a criação e execução de um estudo de caso utilizando o framework apresentado. 
Para a apresentação da pesquisa realizada, o presente artigo é composto por mais cinco seções. A segunda seção apresenta os principais conceitos relacionados à arquitetura multi-tenancy. A terceira, trabalhos relacionados à construção de frameworks que permitem a utilização da arquitetura. A quarta, o framework construído. A quinta, o estudo de caso. Por fim, a sexta apresenta as considerações finais sobre o trabalho e os trabalhos futuros.

\section{Multi-tenancy}

Multi-tenancy é uma arquitetura na qual uma única instância de um aplicativo de software é utilizada por vários consumidores. Cada consumidor é chamado de tenant. Os tenants, cada qual formado por um conjunto de usuários ligados a ele, podem personalizar algumas partes da aplicação, tais como a cor da interface gráfica ou até mesmo regras de negócio, mas estes não podem personalizar o código do aplicativo. Isto é possível porque os dados de cada tenant são logicamente ou fisicamente separados dos demais, ou seja, não é possível para um tenant ter acesso aos dados de outro tenant [Rouse 2011].

A arquitetura multi-tenancy é dividida em três componentes: autenticação, persistência (armazenamento) dos dados em banco de dados e configuração [Bezemer et al. 2010].

\subsection{Autenticação}

Em uma aplicação multi-tenant, todos os tenants utilizam o mesmo ambiente físico, ou seja, compartilham a mesma aplicação e a mesma instância de banco de dados. Para ser capaz de oferecer customização do ambiente e ter certeza de que os tenants podem acessar somente os seus próprios dados, os tenants devem ser autenticados [Bezemer et al. 2010].

Enquanto que a autenticação de usuários é, possivelmente, já presente na aplicação, um componente separado para a autenticação de tenants pode ser necessário. Geralmente é mais fácil introduzir um mecanismo de autenticação adicional, ou seja, remover ou adicionar mais uma tela de login, do que modificar um já existente.

\subsection{Persistência}

Em uma aplicação multi-tenant há uma grande exigência para o isolamento dos dados. Já que todos os tenants usam a mesma instância de um banco de dados, é necessário garantir que eles acessem somente os próprios dados [Bezemer et al. 2010].

Há três formas de isolar os dados utilizando um banco de dados: Máquina Compartilhada, Processo Compartilhado e Tabela Compartilhada [Jacobs e Aulbach 2007].

Na Máquina Compartilhada, cada tenant terá seu próprio banco de dados, porém, compartilhando a mesma infra-estrutura de aplicação. Esta forma é a mais segura das três porque os dados ficam totalmente separados fisicamente e, na ocorrência de um ataque a uma base de dados particular, as demais bases não são afetadas diretamente. Pelo mesmo motivo, essa separação também facilita a migração de dados de seus tenants [Jacobs e Aulbach 2007]. 
A principal limitação desta abordagem é a falta de uso de escalonamento de memória e de disco, afinal, para cada base de dados, a quantidade de memória e de disco utilizada deve ser igualitária e, dependendo do uso por tenant, há desperdício destes, já que um pode necessitar menos que outro [Jacobs e Aulbach 2007].

No Processo Compartilhado, os tenants passam a compartilhar o mesmo banco de dados, consequentemente os mesmos processos e recursos. Apesar de utilizar o mesmo banco de dados, os dados são separados por tabelas, ou seja, cada tenant ainda terá seus dados fisicamente separados dos demais. Além disso, com o compartilhamento de processos e de pool de conexões, o escalonamento de memória é mais eficiente. Sua principal limitação é com relação a flexibilidade de adição e remoção de tenants, porque, para determinados banco de dados, tarefas como remoção em massa de tabelas enquanto o sistema está em execução podem ser problemáticas e comprometer o desempenho [Jacobs e Aulbach 2007].

$\mathrm{Na}$ Tabela Compartilhada, os dados dos tenants são armazenados nas mesmas tabelas, porém cada qual associado com seu respectivo tenant. Para isso é adicionado em cada tabela uma coluna que identifica para qual tenant o dado está associado, ou seja, para cada consulta ao banco de dados esse valor deverá ser informado. Esta forma possui o melhor escalonamento de memória e de disco, porque qualquer operação lida somente com o identificador do tenant em questão [Jacobs e Aulbach 2007].

\subsection{Configuração}

Em uma aplicação multi-tenant, a customização da aplicação deve ser possível através de configuração. Para permitir que o usuário tenha uma experiência como se estivesse trabalhando em um ambiente dedicado, é necessário a implantação de pelo menos quatro tipos de configuração [Bezemer et al. 2010]:

- Estilo de layout: customizar a interface gráfica como temas, logos, banners, e cores.

- Configuração geral: customizar configurações específicas, como chave de criptografia e detalhes do perfil pessoal.

- Entrada e saída de arquivos: customizar a especificação de caminhos para diretório de arquivos, tais como arquivos de relatórios.

- Fluxo de trabalho (Workflow): customizar tarefas que cada tenant pode executar antes e após a realização de uma tarefa comum, como por exemplo, um cadastro de cliente. Como exemplo desta customização pode-se citar o envio de um email personalizado após o cadastro de um usuário na aplicação.

\section{Trabalhos Relacionados}

Já existem algumas formas de implementação de multi-tenancy, dentre elas a fornecida pelo framework Hibernate na sua versão 4. Nessa abordagem, é oferecida uma conexão com o banco de dados que pode variar de acordo com o nome do tenant informado. Essa conexão é a responsável por mapear os tenants e apontar para seus respectivos dados, de forma a abstrair essa implementação do desenvolvedor. Para isso, os tenants ficam associados às conexões e não à lógica de negócio [Red Hat 2013]. 
Isso pode ser considerado um ponto negativo desta abordagem, pelo fato do tenant ser uma extensão de uma tabela que identifica a quem aqueles dados pertencem. Por este motivo, os tenants devem estar associados à quem representa as tabelas na programação orientada a objetos, ou seja, devem estar associados às classes de negócio.

[Bezemer et al. 2010] mostra a construção de um framework que realiza a associação dos tenants às classes de negócio na linguagem .NET, e sugerem a criação de um que promova, além da separação lógica e customização de interface gráfica, a possibilidade de customizar workflows. Para o estudo de caso, são criadas páginas ASP.NET para cada tenant específico para a configuração de interface gráfica. A customização de workflows não foi realizada no estudo de caso.

Utilizando o plugin multi-tenant para Grails, [Neto et al. 2009] associou os tenants à conexão com o banco de dados e também às classes de negócio. Para as classes que são multi-tenant, a anotação@MultiTenant necessita ser declarada. Nem todas as classes precisam ser anotadas, pois nem todas as tabelas de uma aplicação necessitam da separação por tenant, já que podem existir dados que serão compartilhados entre todos os tenants. Como exemplo, pode-se citar uma lista de cidades e estados de um país para a exibição em um cadastro de cliente.

Por conseguinte, com a anotação, é possível identificar quais dados serão restritos e quais serão compartilhados entre os tenants. Em Java, uma anotação é um metadado que fornece dados sobre um programa para serem utilizados em tempo de execução [Oracle 2013].

\section{Framework de Persistência Multi-tenancy}

Esta seção apresenta as características, a estrutura, composições e funcionalidades do framework construído.

\subsection{Especificação do Protótipo}

Com o intuito de explorar os conceitos de multi-tenancy, foi desenvolvido um protótipo de framework em Java capaz de realizar o mapeamento objeto-relacional de entidades de negócio, levando em consideração os tenants criados.

De um modo geral, o framework deve ser capaz de abstrair a complexidade da separação dos dados dos tenants, de uma forma simples, rápida e que exija o mínimo de modificações necessárias para uma possível migração de uma aplicação JDBC singletenant para multi-tenant.

Dentre os modelos de isolamento de dados presentes na literatura sobre multitenancy, o modelo tipo Tabela Compartilhada foi escolhido para ser utilizado pelo framework, por sua facilidade de implementação e eficiência.

Internamente, em nível de banco de dados, a separação dos tenants é dada por uma coluna que irá representá-lo e que é adicionada automaticamente em todas as tabelas que desejam ter seus dados separados. Essa coluna referencia uma nova tabela, chamada de "Tenant", que possui código e nome, criado automaticamente pelo framework. A nível de aplicação, as classes responsáveis pela lógica de negócio e classes de pesquisas ao banco possuem um parâmetro extra que representa o respectivo tenant. Entretanto, suas informações são automaticamente recuperadas em tempo de 
execução pelo framework, para que o desenvolvedor não tenha a necessidade de lidar com este parâmetro.

Para atingir a extensibilidade e fácil adaptação, pensou-se em criar uma camada acima da persistência, abstraída por uma classe de negócio, que realiza as modificações necessárias nas consultas ao banco de dados, facilitando a separação lógica dos dados por tenants. Além disso, os mesmos e as colunas da tabela a serem pesquisadas também foram abstraídas em classes, para organizar a busca dos dados.

Os tenants estão associados somente às classes de negócio e suas respectivas tabelas. Não há a necessidade de associá-los à conexão para realizar a separação dos dados. Na Figura 1 é ilustrada a associação entre o framework, o JDBC e o banco de dados. Observa-se que, para operações multi-tenant, o desenvolvedor não tem contato direto com o JDBC, sendo o framework responsável por abstrair essa comunicação e realizar as operações necessárias.

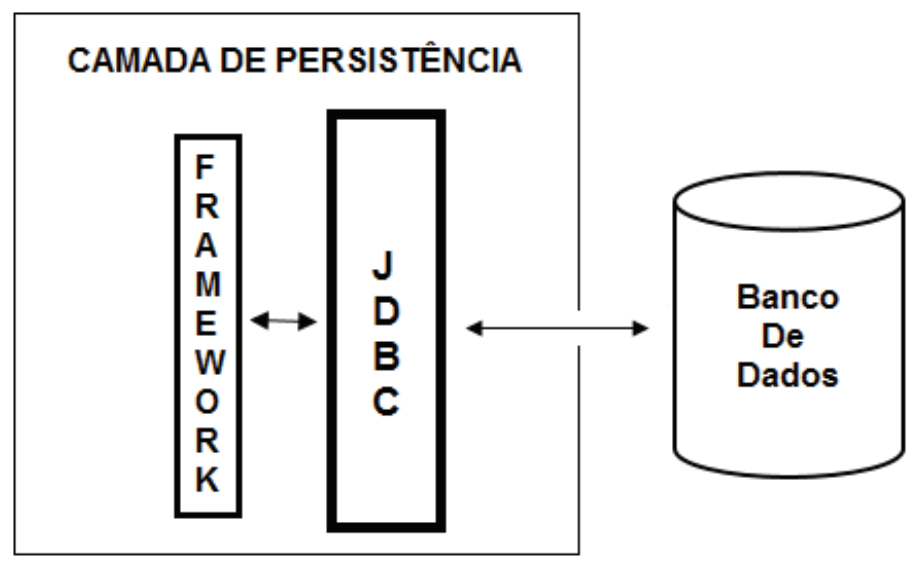

Figura 1. Associação do framework com a API JDBC.

\subsection{Estrutura e Diagrama de Classes}

O framework possui cinco classes, duas interfaces e um enumerator, contendo as operações básicas de Structured Query Language (SQL) sobre uma coluna de uma tabela. Um enumerator é uma lista enumerada de valores pré-definidos [Serson 2009]. Na Figura 2 é ilustrado o diagrama de classes do framework. 


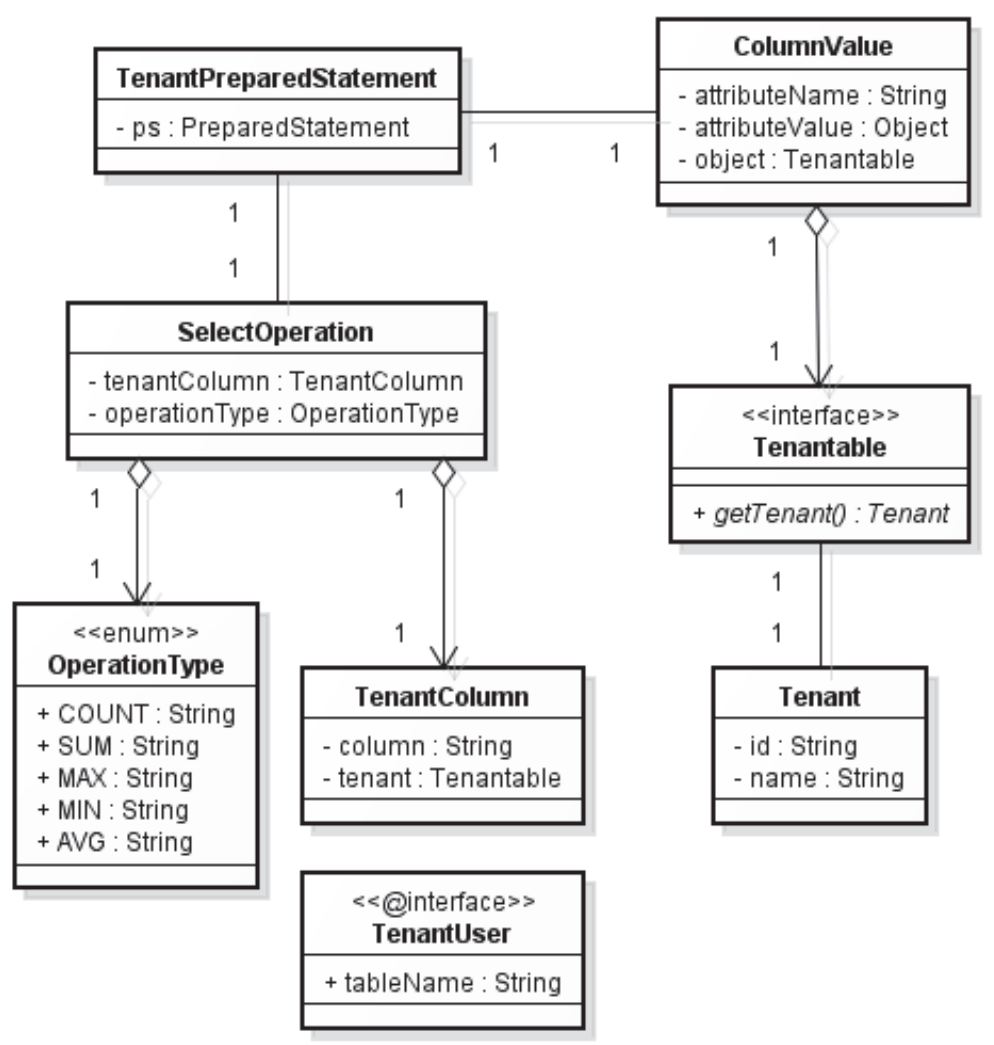

Figura 2. Diagrama de classes do framework.

O relacionamento entre as classes é realizado da seguinte forma:

- A classe TenantPreparedStatement possui relação com a interface PreparedStatement que, por sua vez, é nativa da API JDBC. Esta também possui associação com as classes SelectOperation e ColumnValue que são utilizadas para abstrair dados para as consultas SQL.

- A classe SelectOperation abstrai um TenantColumn e um OperationType, para que seja possível realizar consultas com essas operações de forma correta, simplificada e com um alto nível de abstração.

- O enumerator OperationType armazena o nome algumas operações SQL: Count (Contar elementos), Sum (Somar elementos), Max (Retornar o maior elemento), Min (Retornar o menor elemento) e Avg (Obter a média dos elementos).

- A classe ColumnValue possui relação com dois objetos: um String que representa o nome do atributo a ser utilizado na consulta e um Object que pode assumir qualquer valor a ser buscado para aquele atributo. Ela também possui relação com uma classe de negócio, ou seja, classe da aplicação que irá utilizar o framework, que implementa a interface Tenantable para a comparação de chaves estrangeiras, coluna que faz a associação entre duas ou mais tabelas.

- A interface Tenantable faz uma associação com a classe Tenant. Essa interface deve ser implementada por toda classe de negócio que deverá ter seus dados separados por tenant.

- A classe Tenant representa um tenant, com uma identificação e um nome. 
- A classe TenantColumn possui relação com uma classe que implementa a interface Tenantable e um String que representa a coluna da tabela. É utilizada em conjunto com a classe SelectOperation e também é utilizada na classe TenantPreparedStatement para a operação order by do SQL, que ordena uma consulta por uma determinada coluna da tabela.

- A interface TenantUser é utilizada para definir o nome da tabela da classe que implementa a interface Tenantable. Essa é utilizada também na realização das consultas pela classe TenantPreparedStatement para recuperar esses nomes. Esta interface é declarada como um anotação nas classes de negócio.

A classe base do framework é a TenantPreparedStatement. A partir dela, as consultas são modificadas de forma a considerar os tenants informados e buscar somente os dados pertencentes para aquele tenant. A classe possui oito métodos (funções) públicos que podem ser utilizados, onde um é para criar um tenant e quatro deles se referem à operação select do SQL. Na Figura 3 é ilustrado a entidade TenantPreparedStatement (o nome PreparedStatement foi abreviado para PS a fim de melhorar a visualização).

\begin{tabular}{|c|}
\hline TenantPreparedStatement \\
\hline - ps : PreparedStatement \\
\hline 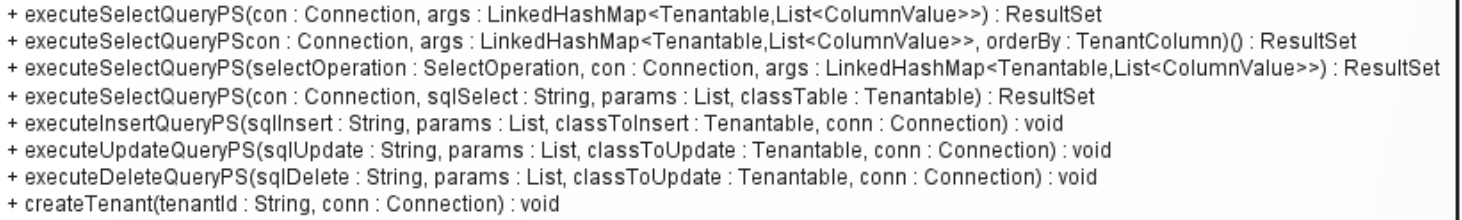 \\
\hline
\end{tabular}

Figura 3. Métodos da classe TenantPreparedStatement.

\section{Estudo de Caso}

A aplicação escolhida para realizar o estudo de caso com o framework criado foi a ONLINESHOP, que simula um site de comércio eletrônico. Esta aplicação possui as opções de adicionar produtos ao carrinho de compras, cadastro de usuários e realização de compras. Sua interface administrativa permite o cadastro, atualização e remoção de produtos, categorias e visualização das vendas realizadas.

A aplicação manipula seis tabelas no banco de dados: a tabela produtos, pedidos, itens_pedido, categorias, usuários e admin, que armazenam, respectivamente, as informações sobre os produtos, tais como id (código de identificação) e nome, informações sobre os pedidos realizados, a associação entre produtos e pedidos, categorias, usuários da aplicação (os consumidores que realizam os pedidos) e os administradores da aplicação (que podem cadastrar os produtos e categorias), como ilustrado na Figura 4. 


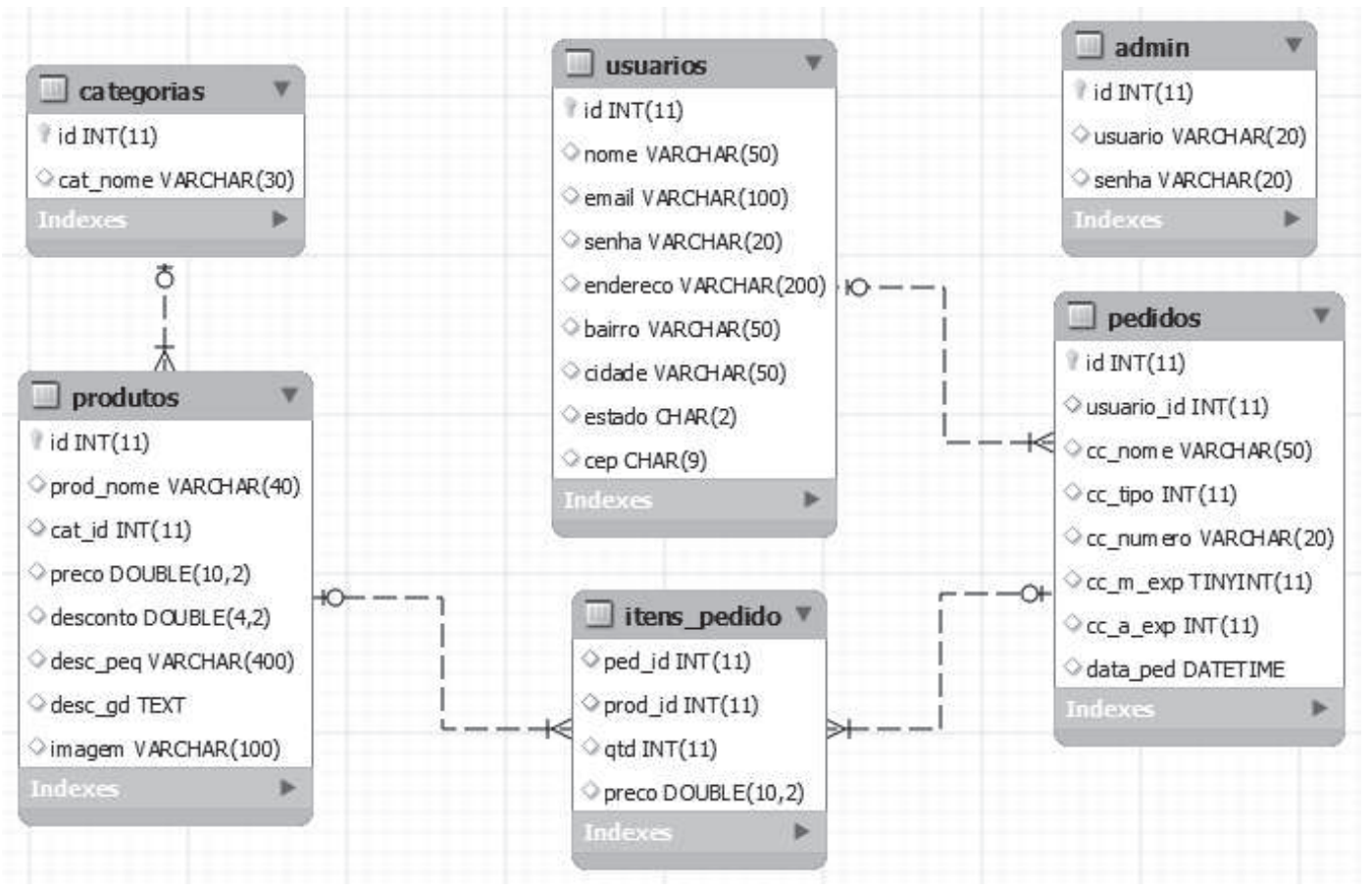

Figura 4. Tabelas manipuladas pela ONLINESHOP.

Após a inserção e utilização do framework, todas as tabelas automaticamente criaram um relacionamento com a nova tabela "tenant", também criada automaticamente por ele. Para complementar, uma nova tabela chamada de "tenantadmin" foi criada manualmente, para a adição (cadastro) de tenants por meio de uma interface gráfica da aplicação e em tempo de execução. O novo relacionamento entre as tabelas é ilustrado na Figura 5.

Observa-se que, para cada tabela da Figura 4, foi adicionado o campo "TENANT_ID", que é o responsável por representar o tenant de cada registro. Como consequência dessa mudança, todos os registros existentes no banco de dados da aplicação foram removidos. Essa atitude evitou que existissem registros sem tenants associados. 


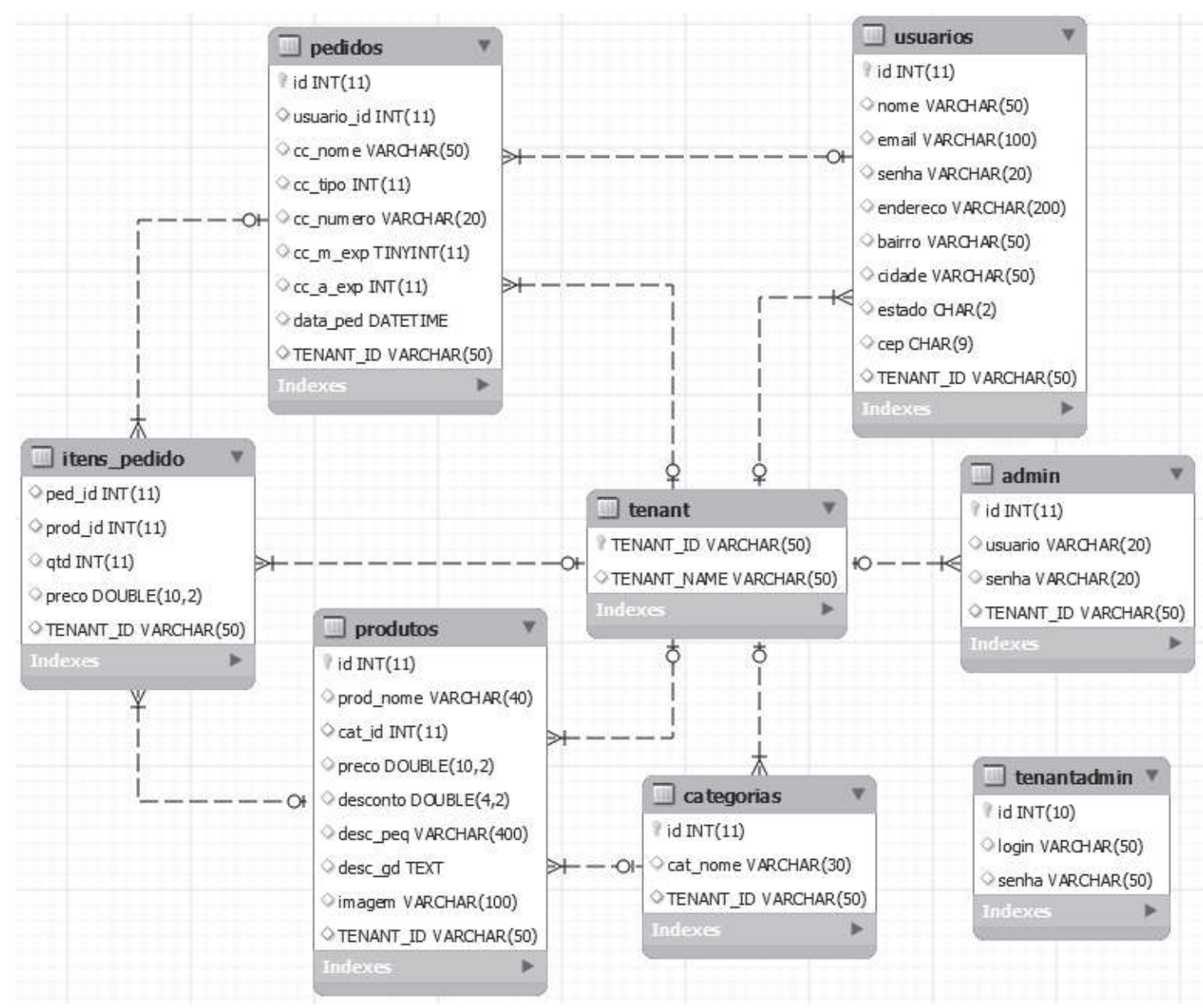

Figura 5. Tabelas da ONLINESHOP após a inserção do framework.

\section{Considerações Finais}

Foi apresentado neste trabalho o conceito de multi-tenancy, uma arquitetura emergente no contexto de desenvolvimento de software, que tem como objetivo permitir que uma mesma aplicação seja acessada por vários usuários diferentes, tendo seus dados, incluindo customizações de interface gráfica e workflows, separados dos demais, dando a impressão que são aplicações diferentes. Também foi apresentada a construção de um framework para a utilização de multi-tenancy com a API JDBC e um estudo de caso para verificar sua aplicabilidade em softwares reais.

O estudo de caso em questão foi uma aplicação de comércio eletrônico chamada ONLINESHOP, que permite exposição de produtos filtrados por categorias, cadastro de usuários que desejam comprar os produtos e a finalização do pedido propriamente dito, originalmente implementada para ser single-tenant. Por ser uma aplicação de comércio eletrônico, a arquitetura multi-tenancy se encaixou facilmente neste contexto, devido à existência de uma grande quantidade de aplicações com esta finalidade e à pouca exigência de mudanças com relação às regras de negócio de cada empresa para este nicho de aplicações.

O framework construído permite que desenvolvedores que ainda utilizam JDBC não necessitem migrar para Hibernate para obter formas mais simples e automatizadas 
de separar dados por tenants, dado que é possível realizar as principais funcionalidades SQL para consulta a banco de dados através do mesmo, embora que seja necessário remover os registros da tabela em que o tenant será inserido, ou migrá-los manualmente, já que cada registro terá que ser associado a um tenant, sendo isto um ponto negativo da utilização do framework.

\subsection{Trabalhos Futuros}

Como trabalhos futuros, propõe-se estudos destinados ao gerenciamento automático de registros que não possuem um tenant associado. Dada a necessidade da remoção dos registros já existentes após a inserção do framework, em uma aplicação single-tenant, ou inclusão manual dos tenants para os registros, na versão atual do framework.

\section{Referências}

Bezemer, P., Zaidman, A., Platzbeecker, B., Hurkmans, T., Hart, A. (2010). Enabling multi-tenancy: An industrial experience report. In Proceedings of the 2010 IEEE International Conference on Software Maintenance, pages 1-8. IEEE.

Brito, E. (2012) "Esqueçam o Service Desk tradicional, agora é SaaS e na nuvem”, http://www.tiinside.com.br/09/04/2012/esquecam-o-service-desk-tradicional-agora-esaas-e-na-nuvem/os/271945/news.aspx, Abril.

Fayad, M. E., Schmidt, D. C., Johnson, R. E. (1999), Building application frameworks: object-oriented foundations of framework design, John Wiley \& Sons.

Jacobs, D. e Aulbach, S. (2007). Ruminations on Multi-Tenant Databases: An industrial experience report. In Proceedings of the Technologie und Web on Datenbanksysteme in Business, pages 514-521. BTW.

Kabanov, J. (2012) "Java EE Productivity Report 2011", http://zeroturnaround.com/rebellabs/java-ee-productivity-report-2011, Novembro.

Neto, J. R., Garcia, V. C., Oliveira, O. S. (2009) "Desenvolvendo aplicações multitenancy para computação em nuvem", http://www.die.ufpi.br/ercemapi2011/minicursos/MC4.pdf, Outubro.

Oracle. (2013) "Lesson: Exceptions", http://docs.oracle.com/javase/tutorial/essential/exceptions, Abril.

Red Hat. (2013) "Chapter 16 Multi-tenancy", http://docs.jboss.org/hibernate/core/4.1/devguide/en-US/html/ch16.html, Abril.

Rouse, M. (2011) “multi-tenancy", http://whatis.techtarget.com/definition/multitenancy, Abril.

Serson, R. R. (2009), Certificação Java 6 - Volume 1 - Teoria, Brasport. 